\title{
Activation of STAT3 stimulates AHSP expression in K562 cells
}

\author{
CAO Cong, ZHAO GuoWei, YU Wei, XIE XueMin, WANG WenTian, YANG RuiFeng, \\ LV Xiang* \& LIU DePei*
}

\begin{abstract}
State Key Laboratory of Medical Molecular Biology, Department of Biochemistry and Molecular Biology, Institute of Basic Medical Sciences, Chinese Academy of Medical Sciences \& Peking Union Medical College, Beijing 100005, China
\end{abstract}

Received October 27, 2013; accepted January 5, 2014; published online April 15, 2014

\begin{abstract}
Studies on the chaperone protein $\alpha$-hemoglobin stabilizing protein (AHSP) reveal that abundant AHSP in erythroid cells enhance the cells' tolerance to oxidative stress imposed by excess $\alpha$-hemoglobin in pathological conditions. However, the potential intracellular modulation of $A H S P$ expression itself in response to oxidative stress is still unknown. The present study examined the effect and molecular mechanism of STAT3, an oxidative regulator, on the expression of AHSP. AHSP expression increased in K562 cells upon cytokine IL-6-induced STAT3 activation and decreased in STAT3 knock-down K562 cells. Regulation of AHSP in oxidative circumstance was then examined in $\alpha$-globin-overloaded K562 cells, and real-time PCR showed strengthened expression of both AHSP and STAT3. ChIP analysis showed binding of STAT3 to AHSP promoter and binding was significantly augmented with IL6 stimulation and upon $\alpha$-globin overexpression. Dual luciferase reporter assays of the wildtype and mutated SB3 element, an IL-6RE site, in the AHSP promoter in K562 cells highlighted the direct regulatory effect of STAT3 on AHSP gene. Finally, direct binding of STAT3 to SB3 site of AHSP promoter was confirmed with EMSA assays. Our work reveals an adaptive AHSP regulation mediated by the redox-sensitive STAT3 signaling pathway, and provides clues to the therapeutic strategy for AHSP enhancement.
\end{abstract}

\section{AHSP, STAT3, oxidative stress, IL6-RE}

Citation: $\quad$ Cao C, Zhao GW, Yu W, Xie XM, Wang WT, Yang RF, LV X, Liu DP. Activation of STAT3 stimulates AHSP expression in K562 cells. Sci China Life Sci, 2014, 57: 488-494, doi: 10.1007/s11427-014-4652-Z

Unbalanced globin chain synthesis exposes and sensitizes erythroid cells to oxidative stress. The newly defined $\alpha$-hemoglobin stabilizing protein (AHSP) is necessary for free $\alpha$-globin stabilization and hemoglobin tetramer assembly $[1,2]$, which guards against the threat imposed by free $\alpha-\mathrm{Hb}$. The interaction between AHSP and $\alpha$-Hb converts the latter into a non-reactive, hexacoordinate ferric form and prevents its damaging effects as a pro-oxidant in vivo, underlying the protective role of $A H S P$ in the redox equilibrium of erythroid cells $[3,4]$. However, how AHSP itself is regulated under oxidative stress has been largely unknown.

As critical signaling molecules under oxidative circumstances, reactive oxygen species (ROS) mediate distinct

*Corresponding author (email: liudp@pumc.edu.cn; lvxiang@pumc.edu.cn) responses to oxidative stress through various pathways $[5,6]$. Signal transducers and activators of transcription (STAT) proteins are a group of important redox regulated effectors [7] that play important roles in regulating hematopoietic homeostasis [8]. STATs can be activated by ROS production in response to the stimulation of cytokines or growth factors, during which STATs are recruited to their cognate receptors and phosphorylated at distinct tyrosine residues by the Janus family of kinases (JAKs) or Src kinases. Activated STATs then homo- or heterodimerize and translocate to the nucleus, initiating a cytoprotective process via modulating the expression of a panel of antioxidant genes or apoptotic genes, and participating in the regulation of cell survival, proliferation and maturation $[9,10]$. STAT3 is a major member of the STATs and is phosphorylated at Tyr705 and 
Ser727 sites upon activation [11]. Constitutive STAT3 phosphorylation exhibits oncogenic properties in promoting cell survival [12], and IL6 (interleukin 6)-gp130-STAT3dependent gene activations have been demonstrated to confer cyto-protection in distinct tissues $[13,14]$.

In this report, we identified the $A H S P$ gene as a STAT3 target. Overloading $\alpha$-globins in K562 cells activates STAT3 and promotes AHSP gene expression. Multiple STAT3 binding sites were detected in the AHSP promoter, among which the SB3 site constitutes a key STAT3 regulating element bordered by a Nrf2/ARE element. Mutation of the consensus cAMP responsive element (CRE) within the Nrf2/ARE element of the SB3 site dramatically decreased STAT3 transactivity on the AHSP promoter and abolished direct binding of STAT3 to the SB3 site.

\section{Materials and methods}

\subsection{Cell culture and drug treatment}

Recombinant IL-6 (SRP3096) was purchased from SigmaAldrich (USA). For IL-6 stimulation, human chronic myelogenous leukemia K562 cells were collected and resuspended in serum free RPMI 1640 medium and treated with IL-6 (100 $\mathrm{ng} \mathrm{mL}{ }^{-1}$ ) for $2 \mathrm{~h}$. The human $\alpha$-globin (HBA) over-expressing K562 stable line was cultured in RPMI 1640 medium containing $10 \%$ fetal bovine serum, and human embryonic kidney $293 \mathrm{~T}$ cells were maintained in Dulbecco's modified Eagle's medium (high sugar) supplemented with $10 \%$ fetal bovine serum.

\subsection{Plasmid construction and transfection}

For generation of over-expression plasmids, the coding sequences of STAT3 and $\alpha$-globin were cloned into pcDNA3.1(-) and pcDNA3.1(+) respectively. STAT3 was knocked down using hairpin RNA directed against the sequence 5'-GCGTCCAGTTCACTACTAA-3', and the oligonucleotides encoding shRNAs were ligated into an RNAi-ready pSIREN-RetroQ vector (Clontech, USA). The resulting STAT3 knock-down K562 cells were generated by retrovirus-mediated RNAi. The pRetroQ-siGFP (5'GCAAGCTGACCCTGAAGTT-3') vector was used as control. The HBA stable line (HBA-K562) and control cells were obtained by transfecting K562 cells with pCDNA3.1HBA-myc and empty vector respectively, using Lipofectamine 2000 (Invitrogen, USA).

\subsection{RNA extraction and real-time qRT-PCR}

Total RNA was extracted from differentially treated K562 cells using Trizol (Invitrogen) according to the manufacturer's instructions. First-strand cDNA was synthesized with M-MLV Reverse Transcriptase and random primer (New England Biolabs, USA). Real-time qPCR experiments were performed using the iQTM5 Real-Time PCR Detection System (Bio-Rad, USA) and SYBRGreen PCR Mix (TaKaRa, Japan). Primer pairs were as follows: AHSP amplication sense 5'-TGATCCTCTCGTCTGAAGAAGAC-3' and antisense 5'-GCTGCCTGTAATAGTTGATGTAGAAGT-3'; and STAT3 amplification: sense 5'-ACCTGCAGCAATACCATTGAC-3' and antisense 5'-AAGGTGAGGGACTCAAACTGC-3'. AHSP and STAT3 expression levels were quantified by normalizing against the internal control $\beta$-actin (sense 5'-GGACATCCGCAAAGACCTGTAC$3^{\prime}$ and antisense 5'-AGGAGGAGCAATGATCTTGATCTT-3') using the delta $\mathrm{Ct}$ method.

\subsection{Western blotting}

The STAT3 knock-down k562 cells were collected and lysed in RIPA buffer (NP-40 0.1\%, NaCl $150 \mu \mathrm{mol} \mathrm{L}^{-1}$, Tris-Cl $10 \mathrm{mmol} \mathrm{L}^{-1}$, NaF $10 \mathrm{mmol} \mathrm{L}{ }^{-1}$, EDTA $2 \mathrm{mmol} \mathrm{L}^{-1}$, DTT $100 \mu \mathrm{g} \mathrm{mL}^{-1}$, PMSF $100 \mu \mathrm{g} \mathrm{mL}^{-1}$ and Protease Inhibitor Cocktail, $\mathrm{pH}$ 8.0). Samples were quantified with a Pierce BCA protein assay kit, separated on a $15 \%$ SDS-PAGE gel and transferred to PVDF membranes. The membranes were then incubated overnight with monoclonal anti-STAT3 (D372G), anti-phospho-STAT3 (Tyr705) from Cell Signaling Technology (USA) or polyclonal anti-GAPDH (FL-335) from Santa Cruz Biotechnology (USA), followed by incubation with corresponding secondary antibodies for approximately $1-2 \mathrm{~h}$ at room temperature. The HBA-K562 stable line was identified with anti-c-Myc (9E10) monoclonal antibodies from Santa Cruz Biotechnology; anti-actin (AC-15) was obtained from Sigma. Bands were visualized with the ECL chemiluminescence kit (Vigorous, China). Human AHSP anti-serum was noncommercial and generously provided by Dr. Weiss (Children's Hospital of Philadelphia, USA).

\subsection{Reporter plasmid construction and luciferase re- porter assay}

The AHSP promoter reporter plasmid (AHSP-Luc) was constructed by PCR amplification of the 1.1 kilobase region (-912 to $+254 \mathrm{bp})$ surrounding AHSP gene transcriptional start site and inserting the PCR product into the MluI/XhoI sites of pGL3-Basic vector (Promega, USA). SB3 reporter plasmids (SB3-Luc or SB3-mut) were constructed by annealing synthesized DNA oligos containing the wild type or mutant STAT3 binding site ( -95 to $-36 \mathrm{bp}$ ) with flanking MluI/XhoI sites, and inserting the resulting double-strand DNA into the pGL3-Enhancer vector (Promega). HEK293T cells were transfected at $50 \%$ confluence using VigoFect according to the manufacturer's instructions (Vigorous). Cells were harvested $48 \mathrm{~h}$ later. Luciferase activity was measured with the Dual Luciferase Reporter Assay System Kit (Promega) and detected in a LB960 Microplate Luminometer (Berthold, Germany). Data were collected in at 
least three separate experiments. The statistical significance test was used to analyze the results.

\subsection{Chromatin immunoprecipitation}

Chromatin immunoprecipitation (ChIP) analysis of control, IL- 6 treated and $\alpha$-globin-overloaded K562 cells was performed as previously described with minor modifications [15]. The precleared cell lysates were immunoprecipitated individually with $4 \mu \mathrm{g}$ of anti-STAT3 (D372G), antiphospho-STAT3 (Tyr705), anti-phospho-STAT3 (Ser727) primary antibodies (Cell Signaling Technology) or $10 \mu \mathrm{L}$ (4 $\mu \mathrm{g}$ ) normal rabbit IgG (Santa Cruz). Precipitated DNA was dissolved in $100 \mu \mathrm{L}$ ddH ${ }_{2} \mathrm{O}$ and analyzed by real-time qPCR using the iQTM5 Real-Time PCR Detection System (Bio-rad) and SYBRGreen PCR Mix (TaKaRa) or by semi-quantitative PCR. PCR primers are listed in Table 1. The $Y$-axis of the quantitative ChIP results is shown as relative enrichment of immunoprecipitated DNA to input. The 5 '-UTR of the gamma-globin gene was used as a positive control for STAT3 binding [16] and the promoter of the brain-specific necdin gene served as a negative control.

\subsection{Electrophoretic mobility shift assay}

Nuclear extracts were prepared from electrophile tBHQ (tert-butylhydroquinone) treated $\left(20 \mu \mathrm{mol} \mathrm{L}{ }^{-1}, 6 \mathrm{~h}\right)$ and control K562 cells as described [17]. EMSA analysis was performed using a LightShift Chemiluminescent EMSA Kit (Pierce, USA) according to the manufacturer's instructions. Each reaction contained $20 \mu \mathrm{L}$ volume including $8 \mu \mathrm{g}$ nuclear extract and $20 \mathrm{nmol} \mathrm{L}{ }^{-1}$ labeled and annealed SB3 probe (forward: biotin-5'-TCTCCTCCCTAGAATGACCTATCACCCTCCTTCAGGACCT-3' and reverse: biotin5'-AGGTCCTGAAGGAGGGTGATAGGTCATTCTAGG GAGGAGA-3') or mutant SB3 probe in which the mutations are shown in lowercase letters (forward: biotin5'-TCTCCTCCCTAGAagacCCTAcagCCCTCCTTCAGGACCT-3' and reverse: biotin-5'-AGGTCCTGAAGGAGGGctgTAGTgtcTTCTAGGGAGGAGA-3'). Nrf2 (4 $\mu \mathrm{g})$ antibody was used in supershift assays, and 150-fold molar excess unlabeled IL-6RE probes (repeated TTCTGGGAA) containing canonic STAT3 binding sequence were pre-incubated in competition experiments. Reaction mixtures were fractionated on non-denaturing $6 \%$ polyacrylamide gels and transferred to a nylon membrane. The DNA was cross-linked to the membrane by UV-light at $150 \mathrm{~mJ}$ $\mathrm{cm}^{-2}$ and the membrane was exposed to X-ray film and developed.

\section{Results}

\subsection{AHSP is a target gene of STAT3}

STAT3 is one of the key regulators in the ability of hema- topoietic cells to cope with oxidative stress [18]. To examine whether the $\alpha$-globin chaperone AHSP is a target gene of STAT3 signaling in redox equilibrium of erythroid cells, we first used cytokine IL-6 as a STAT3 signaling inducer to treat the chronic myelogenous leukemia K562 cell line and detected enhanced AHSP expression after IL-6 treatment (Figure 1A and B). Conversely, knockdown of STAT3 in K562 cells with pSIREN-RetroQ retrovirus expressing short hairpin RNA against STAT3 led to decreased STAT3 as well as AHSP expression at both the mRNA level (Figure 1C) and protein level (Figure 1D). Simultaneous upregulation of STAT3 and AHSP genes were observed in K562 cells overloaded with $\alpha$-globin (HBA-K562), which to some degree mimics the pathologically oxidative environment of $\beta$-thalassemia (Figure $1 \mathrm{E}$ and $\mathrm{F}$ ). These results collectively suggest that STAT3 is a regulator of AHSP gene expression, and implied that AHSP could be targeted by STAT3 in response to oxidative stress in erythroid cells.

\subsection{STAT3 binds to and activates $A H S P$ promoter}

To further explore the regulatory mechanisms through which STAT3 may regulate AHSP expression, we investigated the direct occupancy of STAT3 at the promoter of AHSP in K562 cells. STAT family proteins generally recognize a consensus DNA binding motif of TTCC (C or G) GGAA (or generically TTN5AA) [19]. Three putative STAT3 binding sites (SB), named SB1 to SB3, were predicted in the AHSP promoter (Figure 2A). ChIP analyses were performed to detect the binding of total STAT3 and its active forms, Tyr705 and Ser727 phosphorylated STAT3 (pSTAT3-Tyr705 and pSTAT3-Ser727), at the putative binding sites. In untreated K562 cells, weak binding of STAT3 was detected at the SB1 site, and no binding of either active form of STAT3 was detected. After $2 \mathrm{~h}$ treatment with IL-6 (100 ng mL $\left.{ }^{-1}\right)$, which activates the STAT3 signaling pathway, intense binding of STAT3 was found at SB2 and the pSTAT3-Tyr705 signal became detectable at both SB2 and SB3 sites, while the signal for pSTAT3-Ser727 remained undetectable (Figure 2B). We next examined STAT3 binding status in $\alpha$-globinoverloaded K562 cells. Similar to IL6-induced cells, STAT3 was observed to bind to SB2, while pSTAT3Tyr705was specifically detected at the SB3 site (Figure 2C). No obvious pSTAT3-Ser727 signal was detected.

To examine the transactivity of STAT3 on the AHSP promoter, which contains SB2 and SB3, we established a luciferase reporter construct by inserting the AHSP promoter sequence ( -912 to $+254 \mathrm{bp}$ ) into the pGL3-basic vector (AHSP-Luc). The empty pGL3-basic vector was used as a negative control. Co-expression of STAT3 in HEK293T cells stimulated the activity of AHSP-Luc by nearly 6-fold compared with ASHP-Luc alone, suggesting that STAT3 regulates $A H S P$ expression by targeting its promoter (Figure 2D). 


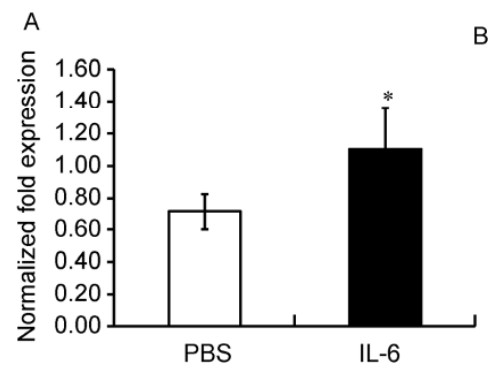

B

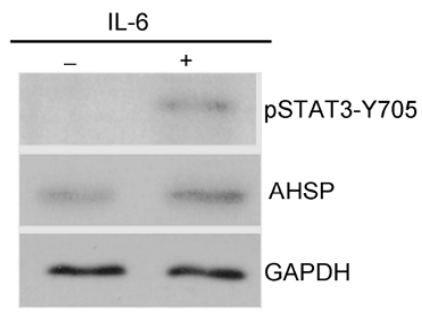

D

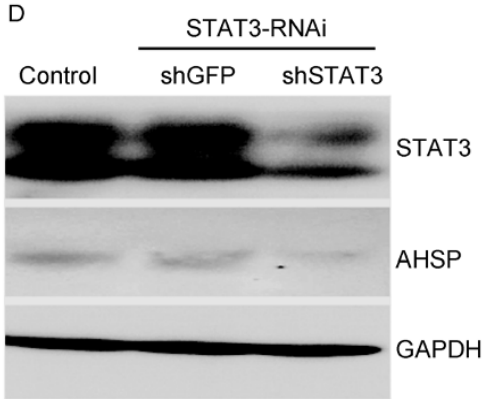

C

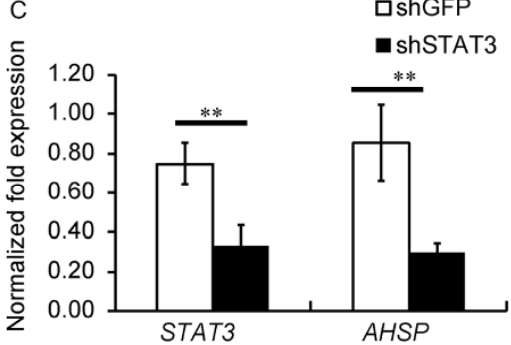

E

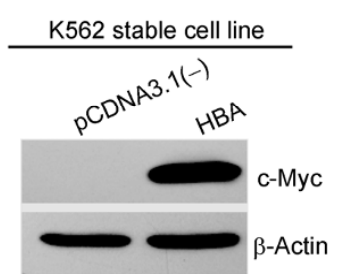

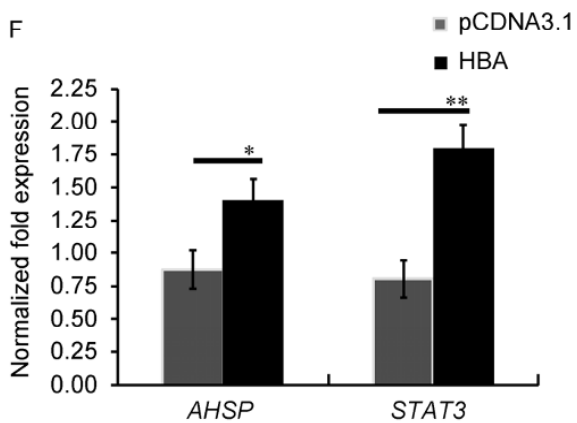

Figure 1 AHSP is a target gene of STAT3. A, Real-time PCR analysis of AHSP expression in IL6-induced (100 ng mL $\left.{ }^{-1}, 2 \mathrm{~h}\right)$ and control K562 cells. B, Western blot analysis of AHSP and p-STAT3(Y705) levels in IL6-induced (100 ng mL $\left.{ }^{-1}, 2 \mathrm{~h}\right)$ and control K562 cells. Real-time PCR (C) and Western blot (D) analysis of AHSP and STAT3 expression in shSTAT3 and control shGFP K562 cells $72 \mathrm{~h}$ after the retrovirus delivery. Untreated K562 cells were used as a negative control in Western blot analysis. E, Western blot analysis of $\alpha$-globin-overloaded and control K562 stable lines. Myc-tagged $\alpha$-globin was identified with anti-myc antibody. F, Real-time PCR analysis of AHSP and STAT3 expression in $\alpha$-globin-overloaded and control K562 stable lines. *, $P<0.05 ; * *, P<0.01$.
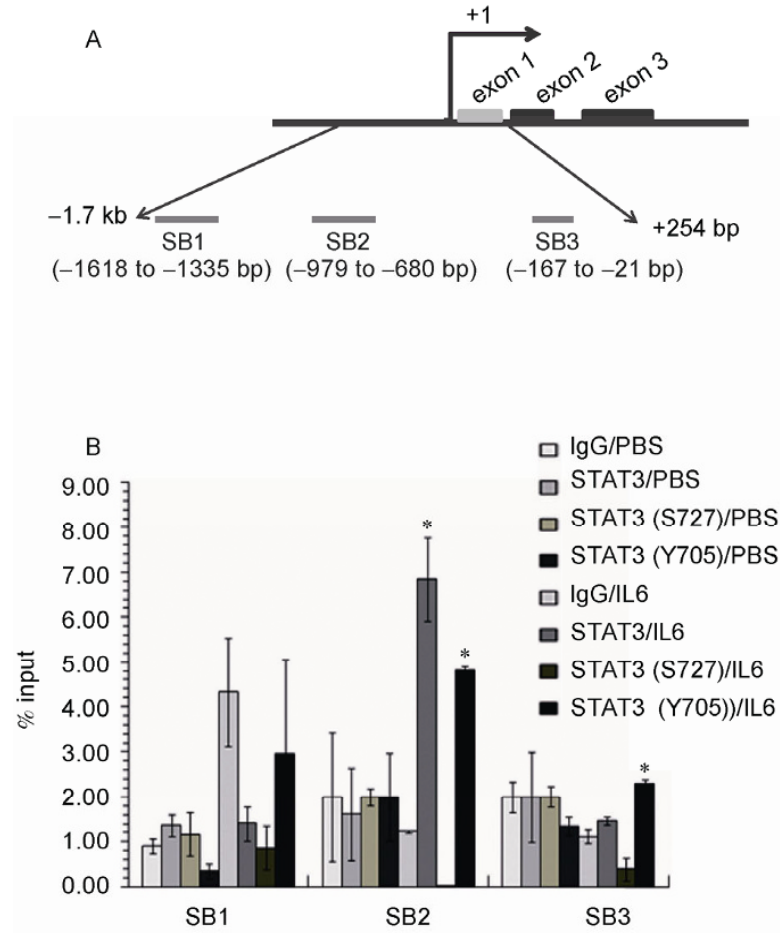
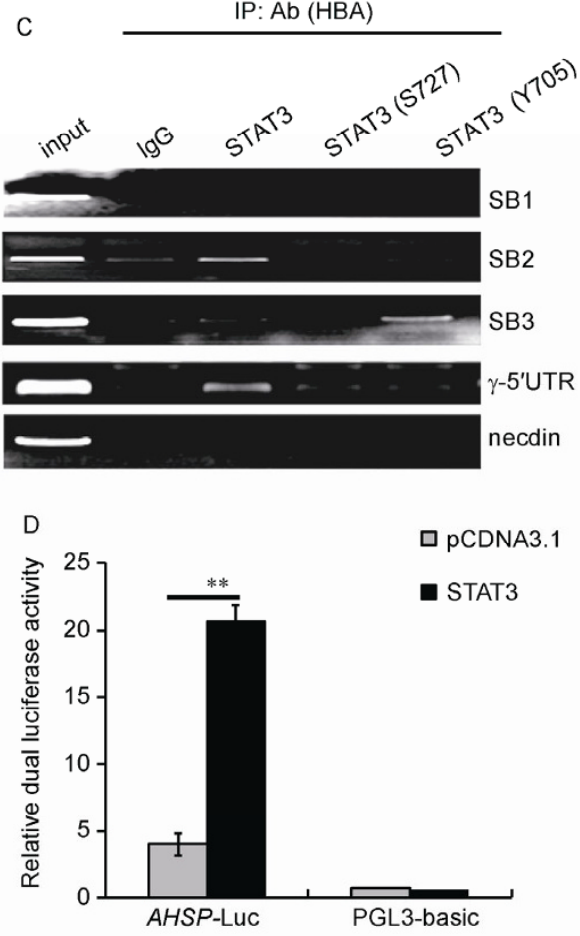

Figure 2 STAT3 binds directly to AHSP promoter region in IL6-stimulated and $\alpha$-globin-overloaded K562 cells. A, The three putative STAT3 binding sites in the AHSP gene promoter. B, Quantitative ChIP analysis of total STAT3 and p-STAT3 occupancy at putative sites in the AHSP promoter in IL6-induced (100 ng mL ${ }^{-1}, 2 \mathrm{~h}$ ) and untreated K562 cells. C, Semi-quantitative ChIP analysis of total STAT3 and p-STAT3 occupancies at putative sites in the AHSP promoter in $\alpha$-globin-overloaded K562 cells. D, Dual luciferase reporter analysis of STAT3 transactivation of AHSP promoter in HEK293T cells. $*, P<0.05$; **, $P<0.01$. 


\subsection{The SB3 element is a critical site for STAT3 bind- ing and activation of $A H S P$ promoter}

In both IL6-induced and $\alpha$-globin-overloaded K562 cells, pSTAT3-Tyr705, the active form of STAT3 was found to strongly bind to the SB3 element ( -167 to $-21 \mathrm{bp}$ ) upstream of the AHSP transcriptional initiation site. Analysis of the SB3 sequence revealed an atypical STAT3/IL-6RE binding site TCCCTAGAA bordered by a cAMP responsive element (CRE)-like element within an atypical Nrf2/ARE site (Figure 3A). The CRE element has been shown to be necessary for STAT3 function in regulating JunB [20] and
STAT3 itself [21]. The luciferase activity of the SB3 reporter (SB3-Luc) was induced by approximately 6-fold with stimulation of STAT3 compared with SB3-Luc alone (Figure 3B). Mutation of TGAC...TCA in the CRE element to GACC...CAG almost completely offset the STAT3 effect (Figure 3B), suggesting the dependency of STAT3 activity on the proximal CRE element.

Direct binding of STAT3 to SB3 element was determined in EMSA analysis (Figure 3C). Two shifted bands were observed with a probe containing the SB3 element, both of which were strengthened with nuclear extracts from
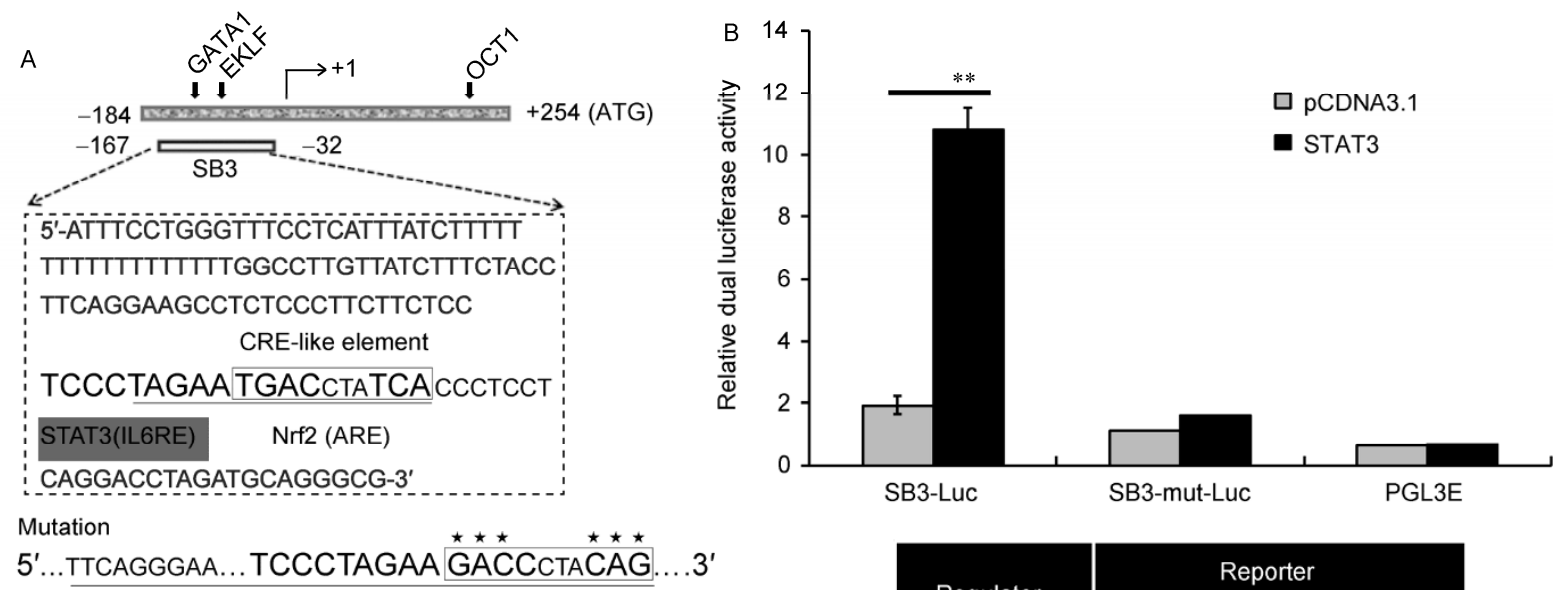

SB3-Luc

\begin{tabular}{|c|c|c|}
\hline \multirow{2}{*}{ Regulator } & \multicolumn{2}{|c|}{ Reporter } \\
\cline { 2 - 3 } & SB3 & SB3-mut \\
\hline STAT3 & 5.57 -fold & 1.42 -fold \\
\hline
\end{tabular}

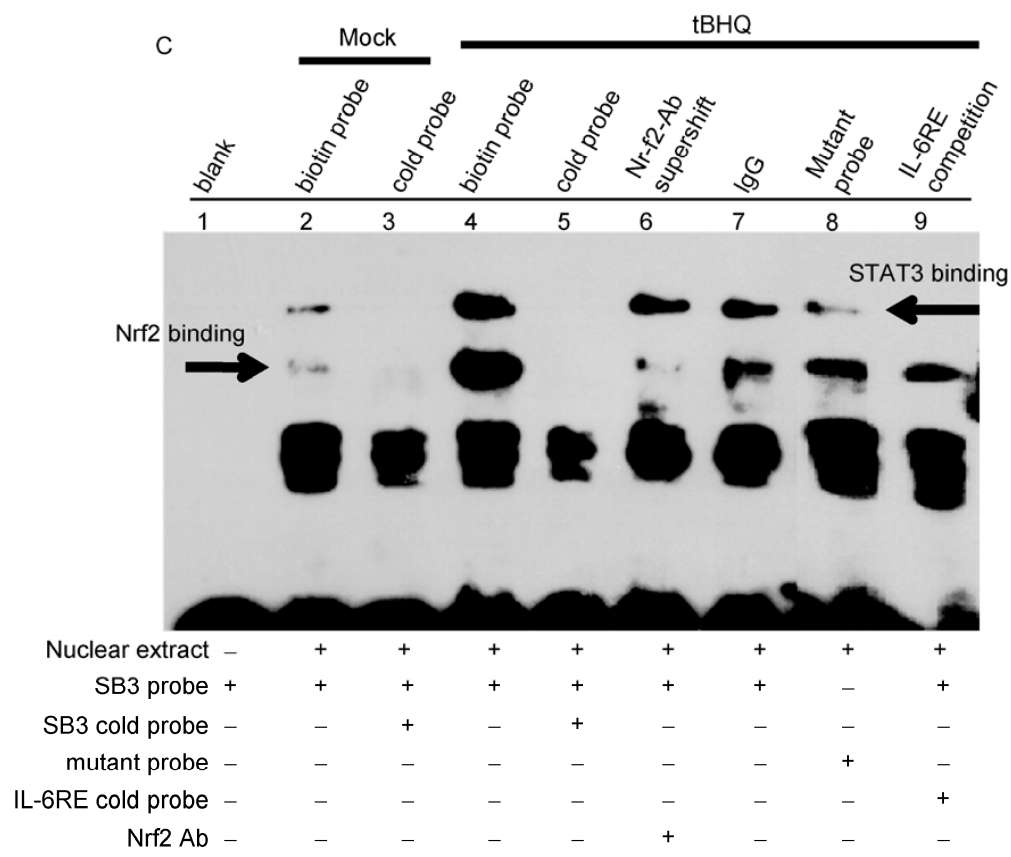

Figure 3 The SB3 element is directly targeted and activated by STAT3. A, Sequence analysis of SB3 in the AHSP proximal promoter. An atypical STAT3 binding site (black capitals) juxtaposed to a putative Nrf2 binding site (underlined) is found in the -61 to -75 bp region of SB3. Consensus CRE-like element (gray box) is shown embedded in the putative Nrf2 binding element. B, Dual luciferase reporter analysis of STAT3 trans-activity on SB3 element in HEK 293 T cells. Mutation of the consensus CRE-like element (labeled with stars) eliminated STAT3 transactivation via the SB3 element. **, $P<0.01$. C, EMSA analysis of the SB3 probe with nuclear extracts from tBHQ treated and control K562 cells. Note that the lower band was specifically supershifted by Nrf2 antibody. Mutation of the CRE-like element had no effect on the lower band, but resulted in the disappearance of the top band, which was completely competed by 150 -fold molar excess of the cold IL-6RE probe. 
tBHQ-treated K562 cells. The lower band was identified as a Nrf2 shift as it was specifically supershifted by Nrf2 antibody. Introduction of the CRE mutation into the SB3 probe resulted in the alleviation of the top shifted band, addition of 150-fold molar excess of cold IL6-RE probe completely competed the shift band away. These observations confirmed the binding of STAT3 on the top band and indicated that the CRE mutation disrupted the binding of STAT3 to the SB3 element.

\section{Discussion}

AHSP is a unique chaperone molecule involved in hemoglobin subunit stabilization and assembly. Lineage specific AHSP expression, like that of many other erythroid specific genes, has been attributed to the master erythroid transcription factors including GATA1, EKLF, and NFE2P45 $[22,23]$. In this study, we show that AHSP expression is directly modulated by STAT3 signaling. We characterized the binding pattern of STAT3 to the AHSP promoter upon IL6 induction and $\alpha$-globin overloading in K562 cells. Furthermore, the CRE element proximal to the STAT3 consensus sequence in the SB3 site of the AHSP promoter was found critical for the binding and activity of STAT3. Our work provides the first evidence for the dynamic regulation of AHSP expression by a cell signaling pathway and implies that AHSP may be an active responder to oxidative stress.

Cytokine IL-6, in combination with multiple factors, is reported to act in the early stage of erythropoiesis [24]. STAT3 is a well-known downstream effector of IL-6 signaling in acute-phase response regulation [25]. It is also shown to be required for erythropoietin receptor dependent regulation of red blood cell development [26]. A recent study claimed that cell-targeted deletion of the STAT3 gene in hematopoietic stem cells/hematopoietic progenitor cells results in excessive ROS production and shortened cell life span [27]. In addition, significant upregulation of STAT3 is found to accompany extramedullary stress erythropoiesis [28], and constitutive STAT3 activation by IL6 has been found in polycythemia vera patients [29]. We show that a $2 \mathrm{~h}$ induction with IL-6 stimulates the binding of pSTAT3Tyr705 to the AHSP promoter and augments AHSP expression in K562 cells, meanwhile, AHSP levels were reduced in STAT3-siRNA K562 cells. Moreover, a concomitant increase of AHSP as well as STAT3 transcription was observed in $\alpha$-globin-overloaded K562 cells. Together, these observations propose AHSP as a new target of STAT3 signaling for orchestrating cellular homeostasis under stress conditions during erythropoiesis. AHSP gene expression was previously reported to increase parallel to $\alpha$-globin during maturation of erythroid cells [30]. The potential involvement of STAT3 remains an intriguing issue to be investigated in primary erythroid cultures or during embryonic hematopoiesis.
Multiple STAT3 binding sites were identified in the AHSP promoter. Although STAT3 knock-down K562 cells showed decreased AHSP expression, the binding of STAT3 in unchallenged K562 cell was weak. Remarkable STAT3 binding was observed after the stimulation by IL-6 or overwhelming free $\alpha$-globin, indicating those sites as predominantly stress-responsive elements. In addition, pSTAT3Tyr705, but not pSTAT3-Ser727 signals, were detected in both IL- 6 treated and $\alpha$-globin-overloaded K562 cells, suggesting divergent functions of these two active STAT3 modifications. The observations are consistent with a previous study which claims that p-STAT3-Tyr705 is necessary for STAT3 activation, while p-STAT3-Ser727 may be asscociated with stronger transactivation [31].

We show that the STAT3 binding site at SB3 is one of the major STAT3 responsive elements in the AHSP promoter. In addition, the SB3 site contains an atypical Nrf2/ARE element intimately connected with the STAT3 site. In addition to the binding of STAT3, EMSA analysis also showed the in vitro binding ability of Nrf2 to SB3. Nrf2 is the central mediator of ROS signaling [32] and has been shown important for maintaining redox balance in hemolyte anemia [33]. Nrf2 also regulates heme synthesis, iron transportation and globin gene expression in erythroid cells $[34,35]$. We found in this work that the Nrf2 activator tBHQ-treated K562 cells nuclear extract displayed apparent increased STAT3-binding to the SB3 site in EMSA. This observation is consistent with the previous study showing that Nrf2 controls the expression of IL-6, which is upstream of STAT3 signaling [36]. Alternatively, this might indicate a potential synergy between Nrf2 and STAT3 when recruiting to target sites in the AHSP promoter. Further investigation of the Nrf2/ARE pathway is therefore necessary for corroborating this promising new candidate in AHSP gene regulation and teasing out the potential cross-talk between Nrf2 and STAT3 in AHSP regulation. Furthermore, how the anti-oxidative response elements, such as the SB3 site identified in this study, may cooperate with the erythroid specific cis-elements [37] in coordinating AHSP expression remains an intriguing issue to be investigated in thalassemia cells.

We thank Professor Mitchell J. Weiss for kindly providing the anti-AHSP antibody. This work was supported by the National Natural Science Foundation of China (31030026, 31021091) and the National Basic Research Program of China (2011CB965203, 2011CB964803).

1 Kihm AJ, Kong YI, Hong W, Russell JE, Rouda S, Adachi K, Simon MC, Blobel GA, Weiss MJ. An abundant erythroid protein that stabilizes free $\alpha$-haemoglobin. Nature, 2002, 417: 758-763

2 Yu X, Kong Y, Dore LC, Abdulmalik O, Katein AM, Zhou S, Choi JK, Gell D, Mackay JP, Gow AJ, Weiss MJ. An erythroid chaperone that facilitates folding of $\alpha$-globin subunits for hemoglobin synthesis. J Clin Invest, 2007, 117: 1856-1865

3 Feng L, Zhou SP, Gu LC, Gell DA, Mackay JP, Weiss MJ, Gow AJ, Shi Y. Structure of oxidized a-haemoglobin bound to AHSP reveals a 
protective mechanism for haem. Nature, 2005, 435: 697-701

4 Mollan TL, Khandros E, Weiss MJ, Olson JS. Kinetics of $\alpha$-globin binding to $\alpha$-hemoglobin stabilizing protein (AHSP) indicate preferential stabilization of hemichrome folding intermediate. J Biol Chem, 2012, 287: 11338-11350

5 Autréaux BD, Toledano MB. ROS as signalling molecules: mechanisms that generate specificity in ROS homeostasis. Nat Rev Mol Cell Biol, 2007, 8: 813-824

6 Trachootham D, Lu WQ, Ogasawara MA, Nilsa RD, Huang P. Redox regulation of cell survival. Antioxid Redox Signal, 2008, 10: 1343-1374

7 Simon AR, Rai U, Fanburg BL, Cochran BH. Activation of the JAK-STAT pathway by reactive oxygen species. Am J Physiol Cell Physiol, 1998, 275: c1640-c1652

8 Rane SG, Reddy EP. JAKs, STATs and Src Kinases in hematopoiesis. Oncogene, 2002, 21: 3334-3358

9 Schindler CW. Series introduction: JAK-STAT signaling in human disease. J Clin Invest, 2002, 109: 1133-1137

10 Menon MP, Karur V, Bogacheva O, Bogachev O, Cuetara B, Wojchowski DM. Signals for stress erythropoiesis are integrated via an erythropoietin receptor-phosphotyrosine-343-Stat5 axis. J Clin Invest, 2006, 116: 683-694

11 Wen ZL, Zhong Z, Darnell JE. Maximal activation of transcription by stat 1 and stat 3 requires both tyrosine and serine phosphorylation. Cell, 1995, 82: 241-250

12 Schuringa JJ, Wierenga AT, Kruijer W, Vellenga E. Consititutive stat3, Tyr705, and Ser727 phosphorylation in acute myeloid leukemia cells caused by the autocrine secretion of interleukin-6. Blood, 2000, 95: 3765-3770

13 Jenkins BJ, Roberts AW, Najdovska M, Grail D, Ernst M. The threshold of gp130-dependent STAT3 signaling is critical for normal regulation of hematopoiesis. Blood, 2005, 105: 3512-3520

14 Hou TY, Tieu BC, Ray S, Recinos Iii A, Cui R, Tilton RG, Brasier AR. Roles of IL-6-gp130 signaling in vascular inflammation. Curr Cardiol Rev, 2008, 4: 179-192

15 Wei GH, Zhao GW, Song W, Hao DL, Lv X, Liu DP, Liang CC. Mechanisms of human $\gamma$-globin transcriptional induction by apicidin involves p38 signaling to chromatin. Biochem Biophys Res Commun, 2007, 363: 889-894

16 Yao X, Kodeboyina S, Liu L, Dzandu J, Sangerman J, Ofori-Acquah SF, Pace BS. Role of STAT3 and GATA1 interactions in $\gamma$-globin gene expression. Exp hematol, 2009, 37: 889-900

17 Zhou LQ, Wu J, Wang WT, Yu W, Zhao GN, Zhang P, Xiong J, Li M, Xue Z, Wang X, Xie XM, Guo ZC, Lv X, Liu DP. The AT-rich DNA-binding protein SATB2 promotes expression and physical association of human $\mathrm{G} \gamma$ - and A $\gamma$-globin genes. J Biol Chem, 2012, 287 30641-30652

18 Wright CJ, Dennery PA. Manipulation of gene expression by oxygen: a primer from bedside to bench. Pediatr Res, 2009, 66: 3-10

19 Darnell JE. STATs and gene regulation. Science, 1997, 277: 1630-1635

20 Kojima H, Nakajima K, Hirano T. IL-6-inducible complexes on an IL-6 response element of the junB promoter contain stat 3 and $36 \mathrm{KD}$ CRE-like site binding protein(s). Oncogene, 1996, 12: 547-554

21 Ichiba M, Nakajima K, Yamanaka Y, Kiuchi N, Hirano T. Autoregulation of the Stat3 gene through cooperation with a cAMP-responsive element-bindding protein. J Biol Chem, 1998, 273: 6132-6138

22 Gallagher PG, Liem RI, Wong E, Weiss MJ, Bodine DM. GATA1 and Oct- 1 are required for expression of the human $\alpha$-hemoglobinstabilizing protein gene. J Biol Chem, 2005, 280: 39016-39023
23 Zhao GW, Yang RF, Lü X, Mitchell WJ, Liu DP, Liang CC. NF-E2: a novel regulator of alpha-hemoglobin stabilizing protein gene expression. Chin Med Sci J, 2010, 25: 193-198

24 Mayani H, Dragowska W, Lansdrop PM. Cytokine-induced selective expansion and maturation of erythroid versus myeloid progenitors from purified core blood precursor cells. Blood, 1993, 81: 3252-3258

25 Gerhartz C, Heesel B, Sasse J, Hemmann U, Landgraf C, Schneider-Mergener J, Horn F, Heinrich PC, Graeve L. Differential activation of acute phase response factor/STAT3 and STAT1 via the cytoplasmic domain of the interleukin 6 signal transducer gp130. J Biol Chem, 1996, 271: 12991-12998

26 Watowich SS, Mikami Aki, Busche RA, Xie X, Pharr PN, Longmore GD. Erythropoietin receptors that signal through Stat5 or Stat3 support fetal liver and adult erythropoiesis: lack of specificity of Stat signals during red blood cell development. J Interf Cytok Res, 2000, 20: $1065-1070$

27 Mantel C, Messina-Graham S, Moh A, Cooper S, Hangoc G, Fu XY, Broxmeyer HE. Mouse hematopoietic cell-targeted STAT3 deletion: stem/progenitor cell defects, mitochondrial dysfunction, ROS overproduction, and a rapid aging-like phenotype. Blood, 2012, 120: 2589-2599

28 Ingley E, McCarthy DJ, Pore JR, Sarna MK, Adenan AS, Wright MJ, Erber W, Tilbrook PA, Klinken SP. Lyn deficiency reduces GATA-1, EKLF and STAT5, and induces extramedullary stress erythropoiesis. Oncogene, 2005, 24: 336-343

29 Röder S, Steimle C, Meinhardt G, Pahl HL. STAT3 is constitutively active in some patients with Polycythemia rubra vera. Exp Hematol, 2001, 29: 694-702

30 Dos Santos CO, Duarte ASS, Saad STO, Costa FF. Expression of $\alpha$-hemoglobin stabilizing protein gene during human erythropoiesis. Exp Hematol, 2004, 32: 157-162

31 Wen ZL. Darnell JE. Mapping of Stat3 serine phosphorylation to a single residue (727) and evidence that serine phosphorylation has no influence on DNA binding of Stat1 and Stat3. Nucl Acids Res, 1997, 25: 2062-2067

32 Mukaigasa K, Nguyen LTP, Li L, Nakajima H, Yamamoto M, Kobayashi M. Genetic evidence of an evolutionarily conserved role for Nrf2 in the protection against oxidative stress. Mol Cell Biol, 2012, 32: 4455-4461

33 Kawatani Y, Suzuki T, Shimizu R, Kelly VP, Yamamoto M. Nrf2 and selenoproteins are essential for maintaining oxidative homeostasis in erythrocytes and protecting against hemolytic anemia. Blood, 2011, 117: 986-996

34 Marro S, Chiabrando D, Messana E, Stolte J, Turco E, Tolosano E, Muckenthaler MU. Heme controls ferroportin1 (FPN1) transcription involving Bach1, Nrf2 and a MARE/ARE sequence motif at position -7007 of the FPN1 promoter. Haematologica, 2010, 95: 1261-1268

35 Macari ER, Lowrey CH. Induction of human fetal hemoglobin via the NRF2 antioxidant response signaling pathway. Blood, 2011, 117: 5987-5997

36 Wruck CJ, Streetz K, Pavic G, Götz ME, Tohidnezhad M, Brandenburg LO, Varoga D, Eickelberg O, Herdegen T, Trautwein C, Cha K, Kan YW, Pufe T. Nrf2 induces interleukin-6(IL-6) expression via antioxidant response element within the IL-6 promoter. J Biol Chem, 2011, 286: 4493-4499

37 Xiong Q, Zhang ZJ, Chang KH, Qu H, Wang H, Qi H, Li Y, Ruan X, Yang Y, Yang Y, Li Y, Sandstrom R, Sabo PJ, Li Q, Stamatoyannopoulos G, Stamatoyannopoulos JA, Fang X. Comprehensive characterization of erythroid- specific enhancers in the genomic regions of human Krüppel-like factors. BMC Genom, 2013, 14: 587

Open Access This article is distributed under the terms of the Creative Commons Attribution License which permits any use, distribution, and reproduction in any medium, provided the original author(s) and source are credited. 